\title{
Diversity-multiplexing tradeoff analysis and optimization for OFDM-based multihop relaying systems
}

\author{
Weiwei Yang ${ }^{1 *}$, Yueming Cai ${ }^{1}$, Wendong Yang $^{1}$ and Baoyu Zheng ${ }^{2}$
}

\begin{abstract}
In this article, we consider a single-antenna orthogonal frequency division multiplexing-based multihop relaying system over frequency selective fading channels employing subcarrier grouping and linear constellation precoding at the source, and linear detection at the destination. We derive closed-form expressions of the diversity-multiplexing tradeoff relation, and propose the optimal precoder design and symbol loading strategy to achieve the optimal diversity performance. Simulation results are presented to verify our theoretical analysis.
\end{abstract}

\section{Introduction}

Multihop relaying communication is well known for being a cost-effective approach to improving the energy efficiency of communication systems in the case of long source-destination distance [1-3]. On the other hand, orthogonal frequency division multiplexing (OFDM) is one of the dominating transmission techniques in many broadband wireless systems [4], e.g., IEEE 802.16 (WiMax), EV-DO Revision C, and the long-term evolution, which divides the broadband wireless channel into a set of orthogonal narrowband subcarriers and hence eliminates the inter-symbol interference. Combining OFDM with multihop relaying has been shown promising in future wireless broadband networks, and has received a lot of attention recently [5-8]. In particular, this OFDM-based multihop relaying architecture has been proposed by current wireless standards, i.e., IEEE 802.16j [5]. Can et al. [6] have considered the implementation issues of OFDM-based multihop cellular networks. The authors of [7] have exploited the hop diversity to improve the spectral efficiency of OFDMbased multihop relaying networks, which employed a decode-and-forward (DF) protocol in each hop link. Zhang et al. [8] further considered OFDM-based linear multihop networks over broadband wireless channels,

\footnotetext{
*Correspondence: yww_1010@yahoo.com.cn

${ }^{1}$ Institute of Communications Engineering, PLA University of Science and

Technology, Nanjing, China

Full list of author information is available at the end of the article
}

and derived the maximum end-to-end average transmission rate and its corresponding optimal power and time allocation. In these prior works, OFDM converts a frequency-selective fading channel into parallel flatfading subchannels and the correlation between adjacent subchannel is ignored for simplicity. However, the price paid for OFDM's simplicity is that these OFDM-based multihop relaying schemes cannot exploit multipath diversity.

In order to exploit multipath diversity, the authors of [9-12] have employed linear constellation precoding for OFDM systems which can be implemented with limited complexity and is especially suitable for high-rate, delay-sensitive applications where powerful outer codes cannot be employed. In most of these works, the decoding at the destination node has been done by using the maximum-likelihood (ML) detector, which has been shown to achieve the maximum possible diversity. However, the ML detector has an exponential complexity in the number of precoded data streams and may not be feasible in practical systems with complexity constraint. The linear detectors, i.e., linear zero-forcing (ZF) detector and linear minimum-mean-square-error (MMSE) detector can reduce the complexity of the receiver, and have been considered in $[11,12]$ for single hop frequency-selective fading channels. However, diversity gain obtained from linear detectors is critically dependent upon the system design,

\section{Springer}

C 2012 Yang et al.: licensee Springer. This is an Open Access article distributed under the terms of the Creative Commons Attribution License (http://creativecommons.org/licenses/by/2.0), which permits unrestricted use, distribution, and reproduction in any medium, provided the original work is properly cited. 
i.e., precoder design, subcarrier grouping, and symbol loading. It is therefore of interest to develop optimal design for OFDM-based multihop relaying systems with linear constellation precoding and linear detection.

The diversity-multiplexing tradeoff (DMT) has originally been developed in [13] to study narrowband single hop multiple-input multiple-output (MIMO) fading channels. Recently, the DMT performance of relay channels under various protocols has been presented in [14-17]. These works mainly focused on two-hop relay networks, where the communication between a source and its destination is assisted by relay nodes. For large networks, multiple hops of relays may be needed to establish communication for a source-destination pair. This multihop relay channel scenario has been investigated in [2,3,18-20]. Sreeram et al. [2,3] have proposed a improved flip-and-forward relay scheme by changing the partitions adaptively according to the multiplexing rates, and observed better DMT performance. In [18], the DMT upper bound for the MIMO multihop channel has been derived by using the capacity cut-set upper bound. In [19], the MIMO multihop relay channel with one multiple-antenna relay at each hop has been studied, and the results showed that under the full-duplex constraint, the DF relay scheme can achieve the DMT cut-set upper bound. The DMT performance of a MIMO full-duplex single-user multihop relay channel has been investigated in [20], and an AF-based space-time relay scheme has been proposed for full-duplex MIMO multihop relay networks to achieve the optimal DMT of the channel. However, these results were discussed mainly for flat-fading channels. The DMT framework has also been used in $[21,22]$ to study frequency-selective fading channels. The fundamental DMT for the MIMO frequencyselective fading channels has been derived in [23] and the code design criteria to achieve this tradeoff was obtained in [24]. However, the design criteria in [24] has been derived assuming the ML detector at the receiver and hence is not applicable to suboptimal linear detectors which are of interest to us. Recently, the authors of [25] have analyzed the DMT performance for multiple-input multiple-output OFDM systems over frequency-selective fading channels. However, to the best of the authors' knowledge, the DMT performance of an OFDM-based multihop relaying system over frequency-selective fading channels remains hitherto unknown, and how to design these OFDM-based relaying systems optimally in terms of DMT is still an open problem, which motivates our work.

In this article, we adopt the framework of DMT to study an OFDM-based multihop relaying system with linear constellation precoding and linear detection over frequency selective fading channels. Our main contributions are summarized in the following.
(1) We derive closed-form expression of the DMT performance for an OFDM-based multihop relaying system employing linear constellation precoding at the source node and linear ZF or MMSE detection at the destination node.

(2) We show that the smallest of the multipath channel orders experienced in each wireless links becomes the performance bottleneck in the high-signal-to-noise ratio (SNR) regime. Different from ML detector, the achievable multipath diversity gain by linear detectors is dependent upon the symbol loading strategy and precoder design for given multihop relaying networks. Furthermore, no diversity gain is achieved when full-rate precoder and linear detector are employed for OFDM-based multihop relaying systems. The loss of multipath diversity is the price paid for simplicity of linear detection.

(3) In order to obtain the maximum multipath diversity gain, we design the optimal symbol loading strategy and give the necessary and sufficient precoder design conditions. Simulation results show that the optimal symbol loading and precoder design strategy can improve the DMT performance significantly.

The rest of this article is organized as follows. In the following section, an OFDM-based multihop relaying system under consideration is briefly described. In Section "DMT analysis", the DMT analysis for the OFDM-based multihop relaying system with linear constellation precoding and linear detection is given. In Section "Optimal transmit strategies design", we develop the optimal transmit strategies for the OFDM-based multihop relaying system, including symbol loading and precoder design. In Section "Simulation results and discussion", some simulation results are presented and discussed. Concluding remarks are given in Section "Conclusions".

Throughout this article, the following notations will be used. $(\cdot)^{T}$ and $(\cdot)^{H}$ denote transpose and Hermitian transpose, respectively. $\operatorname{span}\left\{\mathbf{x}_{1}, \ldots, \mathbf{x}_{k}\right\}$ is the vector subspace spanned by the $k$ vectors $\mathbf{x}_{1}, \ldots, \mathbf{x}_{k} \cdot \operatorname{rank}\{\mathbf{X}\},|\mathbf{X}|$ and $[\mathbf{X}]_{m, n}$ denote the rank, the Frobenius norm, and the element at the $m$ th row and the $n$th column of the matrix $\mathbf{X}$, respectively. vec $\{\mathbf{X}\}$ indicates the vector obtained stacking up the columns of the matrix $\mathbf{X} \cdot \operatorname{diag}\left\{\mathbf{X}_{1}, \ldots, \mathbf{X}_{k}\right\}$ is the block-diagonal matrix containing the matrices $\mathbf{X}_{1}, \ldots, \mathbf{X}_{k}$ on the main diagonal. For any two matrices $\mathbf{A}, \mathbf{B}$ with identical number of rows (columns), $[\mathbf{A}, \mathbf{B}]([\mathbf{A} ; \mathbf{B}])$ denotes the matrix formed by concatenating the columns (rows) of $\mathbf{B}$ with those of $\mathbf{A} . \operatorname{tr}\{\mathbf{A}\}$ and $\lambda_{\min }\{\mathbf{A}\}$ denote the trace and the minimum eigenvalue of the square matrix A. $\mathbf{I}_{k}$ indicates the identity matrix of order $k . \otimes$ denotes the Kronecker product. $E\{\cdot\}$ denotes statistical expectation. $f(\rho) \doteq g(\rho)$ indicates that $\lim _{\rho \rightarrow \infty} \log f(\rho) / \log \rho=$ $\lim _{\rho \rightarrow \infty} \log g(\rho) / \log \rho(\dot{\geq}$ and $\dot{\leq}$ are similarly defined $)$. 


\section{System model}

We consider a wireless multihop OFDM-based relaying system with one source node, one destination node, and $T-1$ relay nodes $(T \geq 2)$, as shown in Figure 1 , where the $t$ th relay is denoted by terminal $t$, and the source and destination are denoted by terminals 0 and $T$, respectively.

We assume that due to the propagation path-loss, the signal transmitted by terminal $t$ can only be received by its direct neighboring nodes, i.e., terminal $t-1$ and terminal $t+1$. Thus, the source signals travel through $T$ hops until they are received by the destination node. Every node is equipped with a single antenna, and we also assume that all relays work in full-duplex mode. This assumption is merely for simplicity of notation. Since we assume that the signal of each node can only be received by its direct neighboring nodes, the half-duplex constraint is directly translated to a reduction of degrees of freedom by a constant factor and does not impact the relaying strategy. The non-regenerative relay strategy is employed at relay nodes, where each relay node simply amplifies and forwards (AF) OFDM symbols. All relay nodes employ amplifiers with fixed gain $\alpha$. Network operators determine the value of $\alpha$.

The channel from terminals $t-1$ to $t(t \in\{1, \ldots, T\})$ is modeled as frequency selective Rayleigh fading and described by the discrete-time impulse response $\mathbf{h}_{t}=$ $\left[h_{t}(0), \ldots, h_{t}\left(L_{t}-1\right)\right]^{T}$, where $L_{t}$ denotes the corresponding channel order, and $h_{t}(l)\left(l \in\left\{0, \ldots, L_{t}-1\right\}\right)$, denotes the Rayleigh fading coefficient of the $l$ th path, and is a zero-mean complex Gaussian random variable with variance $\delta_{t, l}^{2}$. The channel impulse responses include the effects of transmit receive filters, physical multipath, and relative delays among antennas. We assume the channel power constraint as $\sum_{l=0}^{L_{t}-1} \delta_{t, l}^{2}=1$.

At the destination node, after removing the cyclic prefix and applying the FFT, the frequency received signal at the $n$th subcarrier is given by

$$
z(n)=\alpha^{T-1}\left(\prod_{t=1}^{T} H_{t}(n)\right) x(n)+w(n)
$$

where $x(n)$ is the transmitted signal from the source node at the $n$th subcarrier, $E\left\{|x(n)|^{2}\right\}=1, H_{t}(n)$ is the channel frequency response between terminals $t-1$ and $t$ at the $n$th subcarrier. Denote $\mathbf{f}_{L_{t}}(n)=\left[1, e^{-j 2 \pi n / N}, \ldots, e^{-j 2 \pi n\left(L_{t}-1\right) / N}\right]$, and $H_{t}(n)$ can be expressed as

$$
H_{t}(n)=\mathbf{f}_{L_{t}}(n) \mathbf{h}_{t}
$$

The effective noise term $w(n)$ is the noise received at the destination node at the $n$th subcarrier, which captures the overall noise in the multihop channel. Let $v_{t}(n)$ denote the zero-mean complex Gaussian noise with variance $\sigma^{2}$ introduced at the $t$ th hop transmission at the $n$th subcarrier, and $w(n)$ can be expressed as

$$
\begin{aligned}
w(n)= & v_{T}(n)+\alpha H_{T}(n) v_{T-1}(n)+\cdots+\alpha^{T-1} \\
& \times\left(\prod_{i=2}^{T} H_{i}(n)\right) v_{1}(n)
\end{aligned}
$$

Note that $w(n)$ is not white in general. However, since our focus is on the system asymptotic behavior and it has been shown that in this scale of interest, non-Gaussian noise does not affect the analytical results [20]. So, for large $T$, using the central limit theorem, we treat $w(n)$ as Gaussian noise with zero mean and a variance of

$$
E\left\{|w(n)|^{2}\right\} \approx \sigma^{2}\left(1+\sum_{t=1}^{T-1} \alpha^{2 t}\right), \quad \forall n=1, \ldots, N
$$

The destination node normalizes the received signal by a factor of $\sqrt{1 / \sigma^{2}\left(1+\sum_{t=1}^{T-1} \alpha^{2 t}\right)}$. This does not affect

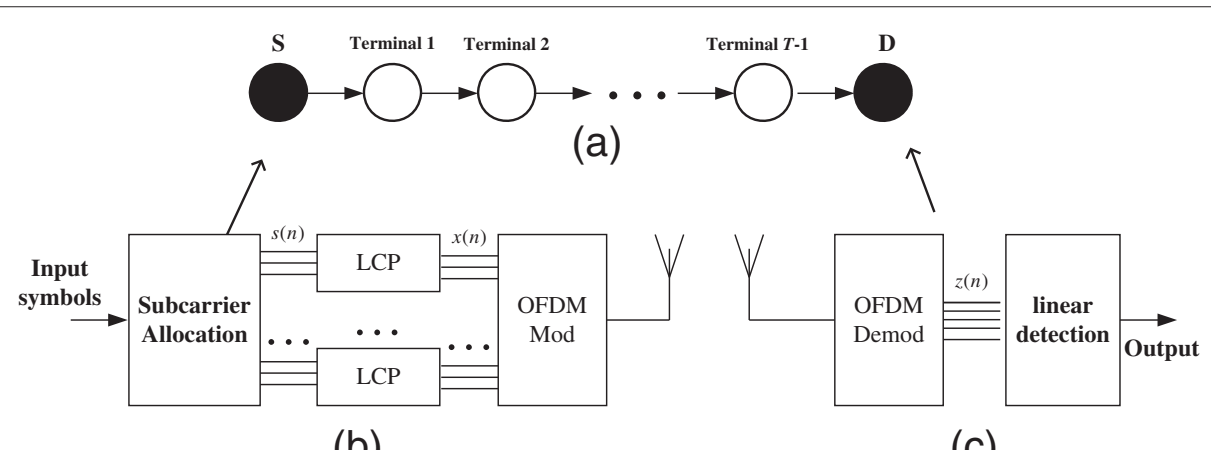

(b)

(c)

Figure 1 (a) Block diagram of the wireless multihop OFDM-based relaying system with one source node, one destination node and T-1 relay nodes. (b) The transmit architecture at $S$ employing subcarrier grouping and linear precoding. (c) The receive architecture at $D$ employing linear detection. 
the SNR, but simplifies the presentation. After normalization, we have

$$
z^{\prime}(n)=\sqrt{\rho}\left(\prod_{t=1}^{T} H_{t}(n)\right) x(n)+w^{\prime}(n)
$$

where $w^{\prime}(n)=w(n) / \sqrt{\sigma^{2}\left(1+\sum_{t=1}^{T-1} \alpha^{2 t}\right)}$ is the zeromean complex Gaussian noise with unit variance, and $\rho=\alpha^{2(T-1)} / \sigma^{2}\left(1+\sum_{t=1}^{T-1} \alpha^{2 t}\right)$ represents the transmit SNR.

\section{Subcarrier grouping and linear constellation precoding}

Subcarrier grouping and linear constellation precoding can exploit the multipath diversity with low-complexity decoding, which has been applied to single-hop or dualhop OFDM systems [9-12]. Each group employs one linear precoder and the groups are independently decoded in parallel. Linear constellation precoding is employed across a group of any $K \leq N$ tones within one OFDM symbol. Let $\mathcal{K} \triangleq\left\{n_{1}, \ldots, n_{K}\right\}$ be the set of index of the precoded tones. Upon defining $\mathcal{H}_{g}=\operatorname{diag}\left(\left[\prod_{t=1}^{T} H_{t}\right.\right.$ $\left.\left.\left(n_{1}\right), \ldots, \prod_{t=1}^{T} H_{t}\left(n_{K}\right)\right]^{T}\right), \mathbf{z}_{g}=\left[z^{\prime}\left(n_{1}\right), \ldots, z^{\prime}\left(n_{K}\right)\right]^{T}, \mathbf{x}_{g}=$ $\left[x\left(n_{1}\right), \ldots, x\left(n_{K}\right)\right]^{T}$, and $\mathbf{w}_{g}=\left[w^{\prime}\left(n_{1}\right), \ldots, w^{\prime}\left(n_{K}\right)\right]^{T}$, the corresponding received signals can be recast as follows:

$$
\mathbf{z}_{g}=\sqrt{\rho} \mathcal{H}_{g} \mathbf{x}_{g}+\mathbf{w}_{g}
$$

where $\mathbf{x}_{g} \triangleq \mathbf{U} \mathbf{s}_{g}, \quad \mathbf{U} \in \mathcal{C}^{K \times M}$ is a linear constellation precoding matrix and $\operatorname{tr}\left\{\mathbf{U} \mathbf{U}^{H}\right\}=K . \mathbf{s}_{g}=\left[s\left(n_{1}\right), \ldots\right.$, $\left.s\left(n_{M}\right)\right]^{T} \in \mathcal{A}^{M}$ is a vector containing $M$ independent and identically distributed information symbols, where $\mathcal{A}$ is the modulation constellation set. $M$ means the number of symbols assigned to each group, which is decided by the symbol loading strategy. We assume $E\left\{\mathbf{s}_{g} \mathbf{s}_{g}^{H}\right\}=\mathbf{I}_{M}$ and the precoding matrix $\mathbf{U}$ is full column-rank, i.e., $\operatorname{rank}(\mathbf{U})=M$.

\section{Linear detection}

The ML decoding complexity increases exponentially with the increase of the size of the constellation alphabet and the column of the precoding matrix $M$, and may not be feasible in practical systems with complexity constraint. To reduce the complexity of the receiver, linear ZF and MMSE detection in [11,12] are employed at the destination. If $\mathcal{H}_{g} \mathbf{U}$ is full column-rank, the ZF detection can be described as

$$
\begin{aligned}
\hat{\mathbf{s}}_{g} & =\left(\mathbf{U}^{H} \mathcal{H}_{g}^{H} \mathcal{H}_{g} \mathbf{U}\right)^{-1} \mathbf{U}^{H} \mathcal{H}_{g}^{H} \mathbf{z}_{g} \\
& =\sqrt{\rho} \mathbf{s}_{g}+\tilde{\mathbf{w}}_{g}
\end{aligned}
$$

where $\tilde{\mathbf{w}}_{g}=\left(\mathbf{U}^{H} \mathcal{H}_{g}^{H} \mathcal{H}_{g} \mathbf{U}\right)^{-1} \mathbf{U}^{H} \mathcal{H}_{g}^{H} \mathbf{w}_{g}$. In this case, $E\left\{\tilde{\mathbf{w}}_{g} \tilde{\mathbf{w}}_{g}^{H}\right\}=\left(\mathbf{U}^{H} \mathcal{H}_{g}^{H} \mathcal{H}_{g} \mathbf{U}\right)^{-1} \triangleq \mathbf{Q}$ and the postprocessing SNR for the $m$ th data symbol is

$$
\mathrm{SNR}_{m}^{Z F}=\frac{\rho}{[\mathbf{Q}]_{m, m}}
$$

The MMSE detection can be described as

$$
\hat{\mathbf{s}}_{g}=\left(\mathbf{U}^{H} \mathcal{H}_{g}^{H} \mathcal{H}_{g} \mathbf{U}+\frac{1}{\rho} \mathbf{I}_{M}\right)^{-1} \mathbf{U}^{H} \mathcal{H}_{g}^{H} \mathbf{z}_{g}
$$

In this case, the post-processing SNR for the $m$ th data symbol is

$$
\mathrm{SNR}_{m}^{\mathrm{MMSE}}=\frac{\rho}{\left[\left(\mathbf{U}^{H} \mathcal{H}_{g}^{H} \mathcal{H}_{g} \mathbf{U}+\frac{1}{\rho} \mathbf{I}_{M}\right)^{-1}\right]_{m, m}}-1
$$

As stated in [26], in the following we assume that the residual interference plus noise at the output of the MMSE filter can be modeled as Gaussian random variable.

\section{DMT analysis}

In this section, we study the DMT relation of an OFDMbased multihop relaying system over frequency selective fading channels, which employs subcarrier grouping and linear constellation precoding at the source and linear ZF or MMSE detection at the destination.

Let $\mathcal{E}$ indicate the event that at least one of the entries of $\mathbf{s}_{g}$ is erroneously detected and $R(\rho)$ be the transmit rate over a group of $K$ tones. We say that a transmission scheme achieves a spatial multiplexing gain of $\gamma_{g}$ if the supported data rate

$$
R(\rho) \approx \gamma_{g} \log \rho
$$

where $\gamma_{g} \in[0, K]$ denotes the multiplexing gain over a group of $K$ tones. The DMT can be characterized by mapping the diversity gain as a function of $\gamma_{g}$, i.e., $d\left(\gamma_{g}\right)$, where $d\left(\gamma_{g}\right)$ is the diversity gain and defined by

$$
d\left(\gamma_{g}\right)=\lim _{\rho \rightarrow \infty}-\frac{\log (\operatorname{Pr}(\mathcal{E}))}{\log \rho}
$$

where $\operatorname{Pr}(\mathcal{E})$ is the probability of the event $\mathcal{E}$.

Let $\gamma_{g} \in[0, K]$ denote the multiplexing gain over a group of $K$ subcarriers. The next theorem analyzes the DMT performance of the OFDM-based multihop relaying system.

Theorem 1 Employing a given full column-rank precoder $\mathbf{U} \in \mathcal{C}^{K \times M}$ to implement linear constellation precoding over an arbitrary group of $K \leq N$ tones at the source node and linear ZF detection at the destination node, the DMT of the OFDM-based multihop relaying system is

$$
d\left(\gamma_{g} ; M ; G ; K\right)= \begin{cases}\left(1-\gamma_{g} / M\right) \min \left\{K-G+1, L_{\min }\right\}, & \gamma_{g} \in[0, M] \\ 0 & , \gamma_{g} \in[M, K]\end{cases}
$$


Proof of Theorem 1 Consider first the ZF detector. Based on (11), we have

$$
\begin{aligned}
\operatorname{Pr}(\mathcal{E}) & =\operatorname{Pr}\left\{\bigcup_{m=1}^{M}\left(\log \left(1+\mathrm{SNR}_{m}^{\mathrm{ZF}}\right)<\frac{1}{M} R(\rho)\right)\right\} \\
& \doteq \operatorname{Pr}\left\{\bigcup_{m=1}^{M}\left(\mathrm{SNR}_{m}^{Z F}<\rho^{\gamma}\right)\right\} \\
& =\operatorname{Pr}\left\{\bigcup_{m=1}^{M}\left(\frac{1}{[\mathbf{Q}]_{m, m}}<\rho^{-(1-\gamma)}\right)\right\} \\
& =\operatorname{Pr}\left\{\frac{1}{\max _{m}\left([\mathbf{Q}]_{m, m}\right)}<\rho^{-(1-\gamma)}\right\}
\end{aligned}
$$

where $\gamma=\gamma_{g} / M$. Due to employing the linear constellation precoding matrix $\mathbf{U}$, we can transmit $M$ parallel data streams over a group of $K$ tones. Furthermore, it exploit the correlation structure of the OFDM subchannels and can achieve multipath diversity gain.

Notice that $\max \left\{[\mathbf{Q}]_{m, m}\right\} \leq \operatorname{tr}\{\mathbf{Q}\} \leq M \max \left\{[\mathbf{Q}]_{m, m}\right\}$, and (14) can be expressed as

$$
\operatorname{Pr}(\mathcal{E}) \doteq \operatorname{Pr}\left\{\frac{1}{\operatorname{tr}(\mathbf{Q})}<\rho^{-(1-\gamma)}\right\}
$$

Considering that $\left\{\mathbf{h}_{t}\right\}_{t=1}^{T}$ are independent Gaussian random vectors, and $\operatorname{rank}(\mathbf{U})=M$, it is clear that $\operatorname{Pr}\left\{\operatorname{rank}\left(\mathcal{H}_{g} \mathbf{U}\right)=M\right\}=1$. So, we know that $\lambda_{\text {min }}$ $\left(\mathbf{U}^{H} \mathcal{H}_{g}^{H} \mathcal{H}_{g} \mathbf{U}\right)>0$ with probability one. Using the property of $\frac{1}{\lambda_{\min }\left(\mathbf{U}^{H} \mathcal{H}_{g}^{H} \mathcal{H}_{g} \mathbf{U}\right)} \leq \operatorname{tr}(\mathbf{Q}) \leq \frac{M}{\lambda_{\min }\left(\mathbf{U}^{H} \mathcal{H}_{g}^{H} \mathcal{H}_{g} \mathbf{U}\right)}$, can be rewritten as

$$
\operatorname{Pr}(\mathcal{E}) \doteq \operatorname{Pr}\left\{\lambda_{\min }\left(\mathbf{U}^{H} \mathcal{H}_{g}^{H} \mathcal{H}_{g} \mathbf{U}\right)<\rho^{-(1-\gamma)}\right\}
$$

Using the Rayleigh-Ritz Theorem [27, Theorem 4.2.2], we have

$$
\lambda_{\min }\left(\mathbf{U}^{H} \mathcal{H}_{g}^{H} \mathcal{H}_{g} \mathbf{U}\right) \leq \mathbf{a}^{H} \mathbf{U}^{H} \mathcal{H}_{g}^{H} \mathcal{H}_{g} \mathbf{U a}
$$

where $\mathbf{a} \in C^{M}$ is any unit norm vector.

Considering that rank $(\mathbf{U})=M$, and let $\mathbf{u}_{i}^{H}$ be the $i$ th row of $\mathbf{U}=\left[\mathbf{u}_{1}, \ldots, \mathbf{u}_{K}\right]^{H}$, we can define as [22]

$$
\begin{aligned}
G & \triangleq \min _{M \leq i \leq K}\left\{i: \operatorname{rank}\left\{\left[\mathbf{u}_{k_{1}}, \ldots, \mathbf{u}_{k_{i}}\right]^{H}\right\}=M,\right. \\
\forall k_{1} & \left.\neq k_{2} \ldots \neq k_{i}, k_{j} \in\{1, \ldots, K\}\right\}
\end{aligned}
$$

By definition, any set of $G$ rows of $U$ spans $\mathcal{C}^{M}$ and there exists at least one set of $G-1$ rows of $U$ which lies in an $M-1$-dimensional subspace of $\mathcal{C}^{M}$. Without loss of generality, let us assume here $\operatorname{rank}\left\{\left[\mathbf{u}_{1}, \ldots, \mathbf{u}_{G}\right]^{H}\right\}=M$ and $\operatorname{rank}\left\{\left[\mathbf{u}_{1}, \ldots, \mathbf{u}_{G-1}\right]^{H}\right\}=M-1$. If we select $\mathbf{a} \in \mathcal{U}^{\perp}$ where $\mathcal{U}=\operatorname{span}\left(\mathbf{u}_{1}, \ldots, \mathbf{u}_{G-1}\right)$, we have $c_{j}=\left|\mathbf{u}_{j}^{H} \mathbf{a}\right|^{2}=$ 0 for $j \in\{1, \ldots, G-1\}$ and $c_{j}=\left|\mathbf{u}_{j}^{H} \mathbf{a}\right|^{2} \neq 0$ for $j \in\{G, \ldots, K\}$. Notice that the non-negative constant $c_{j}$ is independent of the channel and $\rho$. Let $c_{\max }=\max _{j}\left\{c_{j}\right\}$, for any $1 \leq M \leq K$, we can obtain

$$
\begin{aligned}
\lambda_{\min }\left(\mathbf{U}^{H} \mathcal{H}_{g}^{H} \mathcal{H}_{g} \mathbf{U}\right) & \leq \mathbf{a}^{H} \mathbf{U}^{H} \mathcal{H}_{g}^{H} \mathcal{H}_{g} \mathbf{U a} \\
& \leq c_{\max } \sum_{j=G}^{K}\left|\prod_{t=1}^{T} H_{t}\left(n_{j}\right)\right|^{2} \\
& =c_{\max }\left|\mathbf{h}_{G}\right|^{2}
\end{aligned}
$$

where $\mathbf{h}_{G}=\left[\prod_{t=1}^{T} H_{t}\left(n_{G}\right), \ldots, \prod_{t=1}^{T} H_{t}\left(n_{K}\right)\right]^{T}$.

The probability $\operatorname{Pr}(\mathcal{E})$ can be lower-bounded by

$$
\begin{aligned}
\operatorname{Pr}(\mathcal{E}) & \doteq \operatorname{Pr}\left\{c_{\max }\left|\mathbf{h}_{G}\right|^{2}<\rho^{-(1-\gamma)}\right\} \\
& \doteq \operatorname{Pr}\left\{\left|\mathbf{h}_{G}\right|^{2}<\rho^{-(1-\gamma)}\right\}
\end{aligned}
$$

Without loss of generality, let $L_{t_{1}} \leq L_{t_{2}} \leq \cdots \leq L_{t_{T}}$ where $t_{1}, \ldots, t_{T}$ are the index of the ordered $T$ hop channels according to their channel orders. So, the channel with the smallest order is $\mathbf{h}_{t_{1}}$ with the order $L_{\text {min }} \triangleq$ $\min \left\{L_{1}, \ldots, L_{T}\right\}=L_{t_{1}}$. Then, repeating to apply the property of vec $(\mathbf{A B C})=\left(\mathbf{C}^{T} \otimes \mathbf{A}\right) \operatorname{vec}(\mathbf{B})$, we can rewrite $\mathbf{h}_{G}$ as

$$
\mathbf{h}_{G}=\boldsymbol{\Psi} \prod_{j=2}^{T}\left\{\mathbf{I}_{\left(\prod_{i=1}^{j-1} L_{t_{i}}\right)} \otimes \mathbf{h}_{t_{j}}\right\} \mathbf{h}_{t_{1}}
$$

where $\boldsymbol{\Psi}=\left[\boldsymbol{\kappa}_{n_{G}}^{T}, \ldots, \boldsymbol{\kappa}_{n_{K}}^{T}\right]^{T}, \boldsymbol{\kappa}_{n}=\mathbf{f}_{L_{t_{1}}}(n) \prod_{j=2}^{T}\left\{\mathbf{I}_{\left(\prod_{i=1}^{j-1} L_{t_{i}}\right)}\right.$ $\left.\otimes \mathbf{f}_{t_{j}}(n)\right\}$.

Denote $\boldsymbol{\Phi}=\left(\prod_{j=2}^{T}\left\{\mathbf{I}_{\left(\prod_{i=1}^{j} L_{t_{i}}\right)} \otimes \mathbf{h}_{t_{j}}\right\}\right)^{H} \boldsymbol{\Psi}^{H} \boldsymbol{\Psi}\left(\prod_{j=2}^{T}\right.$ $\left.\left\{\mathbf{I}_{\left(\prod_{i=1}^{j} L_{t_{i}}\right)} \otimes \mathbf{h}_{t_{j}}\right\}\right)$, and we have

$$
\operatorname{Pr}(\mathcal{E}) \dot{\operatorname{Pr}}\left\{\mathbf{h}_{t_{1}}^{H} \boldsymbol{\Phi} \boldsymbol{h}_{t_{1}}<\rho^{-(1-\gamma)}\right\}
$$

Notice that $\operatorname{rank}\{\boldsymbol{\Phi}\}=\min \left\{K-G+1, L_{\min }\right\} \triangleq \eta$, and let $\beta_{\min }^{2}$ be the minimum nonzero eigenvalue of $\boldsymbol{\Phi}$ and $\mathbf{h}=$ $\left[h_{t_{1}}(0), \ldots, h_{t_{1}}\left(\eta_{2}-1\right)\right]^{T}$. Then, we can get

$$
\operatorname{Pr}(\mathcal{E}) \dot{\operatorname{Pr}}\left\{\beta_{\min }^{2} \mathbf{h}^{H} \mathbf{h}<\rho^{-(1-\gamma)}\right\}
$$

Define $\varphi=\sum_{l=1}^{\eta}\left|h_{t_{1}}(l)\right|^{2}$, and $\varphi$ is a Chi-squared random variable with $2 \eta$ degrees of freedom. Hence, we can obtain the lower bound of $\operatorname{Pr}(\mathcal{E})$

$$
\begin{aligned}
\operatorname{Pr}(\mathcal{E}) & \geq \operatorname{Pr}\left\{\varphi<\rho^{-(1-\gamma)} / \beta_{\min }^{2}\right\} \\
& \doteq \rho^{-(1-\gamma) \min \left\{K-G+1, L_{\min }\right\}}
\end{aligned}
$$


We now derive the upper bound of $\operatorname{Pr}(\mathcal{E})$. We can always reorganize tones such that $\mathbf{U}=\left[\mathbf{U}_{1} ; \mathbf{U}_{2}\right], \mathcal{H}_{g}=$ $\operatorname{diag}\left\{\mathcal{H}_{g 1}, \mathcal{H}_{g 2}\right\}$, and

$$
\mathbf{U}^{H} \mathcal{H}_{g}^{H} \mathcal{H}_{g} \mathbf{U}=\mathbf{U}_{1}^{H} \mathcal{H}_{g 1}^{H} \mathcal{H}_{g 1} \mathbf{U}_{1}+\mathbf{U}_{2}^{H} \mathcal{H}_{g 2}^{H} \mathcal{H}_{g 2} \mathbf{U}_{2}
$$

where $\mathbf{U}_{1} \in \mathcal{C}^{M \times M}, \mathbf{U}_{2} \in \mathcal{C}^{(K-M) \times M}, \mathcal{H}_{g 1} \in \mathcal{C}^{M \times M}$, and $\mathcal{H}_{g 2} \in \mathcal{C}^{(K-M) \times(K-M)}$. In the following, we collect in $\mathcal{H}_{g 2}^{H} \mathcal{H}_{g 2}$ the $K-G$ tones with the worst value of $\left|H_{S R}\left(n_{j}\right) H_{R D}\left(n_{j}\right)\right|^{2}$. Also, among the remaining $G$ tones, we include in $\mathcal{H}_{g 2}^{H} \mathcal{H}_{g 2}$ the set of $G-M$ tones which makes $\mathbf{U}_{1}$ full rank. Consider that $\mathbf{U}_{2}^{H} \mathcal{H}_{g 2}^{H} \mathcal{H}_{g 2} \mathbf{U}_{2}$ is Hermitian and positive semi-definite, and use the Weyl theorem [27, Corollary 4.3.3], we can obtain

$$
\begin{aligned}
\operatorname{Pr}(\mathcal{E}) & \doteq \operatorname{Pr}\left\{\lambda_{\min }\left(\mathbf{U}^{H} \mathcal{H}_{g}^{H} \mathcal{H}_{g} \mathbf{U}\right)<\rho^{-(1-\gamma)}\right\} \\
& \doteq \operatorname{Pr}\left\{\lambda_{\min }\left(\mathbf{U}_{1}^{H} \mathcal{H}_{g 1}^{H} \mathcal{H}_{g 1} \mathbf{U}_{1}\right)<\rho^{-(1-\gamma)}\right\} \\
& \doteq \operatorname{Pr}\left\{\lambda_{\min }\left(\mathbf{U}_{1}^{H} \mathbf{U}_{1}\right) \lambda_{\min }\left(\mathcal{H}_{g 1}^{H} \mathcal{H}_{g 1}\right)<\rho^{-(1-\gamma)}\right\}
\end{aligned}
$$

where the second inequality employs the Ostrowski theorem [27, Theorem 4.5.9]. Finally, notice that $\lambda_{\min }\left(\mathbf{U}_{1}^{H} \mathbf{U}_{1}\right)$ depends upon the specific tone ordering employed in (25); nevertheless, we can always find a positive constant $C_{2}$ (which is independent of the channel and $\rho$ ) such that $\lambda_{\min }\left(\mathbf{U}_{1}^{H} \mathbf{U}_{1}\right) \geq C_{2}$ for all possible tone orderings. So, (26) can be expressed as

$$
\operatorname{Pr}(\mathcal{E}) \dot{\leq} \operatorname{Pr}\left\{\lambda_{\min }\left(\mathcal{H}_{g 1}^{H} \mathcal{H}_{g 1}\right)<\rho^{-(1-\gamma)}\right\}
$$

Let $\tau=K-G+1$ and $\left\{\mathfrak{s}_{i}\right\}$ indicate all possible sets of $K-G+1$ elements out of $\left\{n_{1}, \ldots, n_{K}\right\}, i=1, \ldots, \xi$, $\xi=\left(\begin{array}{c}K \\ \tau\end{array}\right)$. If $\lambda_{\min }\left(\mathcal{H}_{g 1}^{H} \mathcal{H}_{g 1}\right)<\rho^{-(1-\gamma)}$, it means that there have at least $\tau$ distinct tone index in deep fade. So, we have

$$
\begin{aligned}
\operatorname{Pr}(\mathcal{E}) & =\operatorname{Pr}\left\{\underset{i=1, \ldots, \xi}{\mathrm{U}}\left(\bigcap_{\alpha \in \Im_{i}}\left|\prod_{t=1}^{T} H_{t}(\alpha)\right|^{2}<\rho^{-(1-\gamma)}\right)\right\} \\
& \leq \sum_{i=1, \ldots, \xi} \operatorname{Pr}\left\{\bigcap_{\alpha \in \Im_{i}}\left|\prod_{t=1}^{T} H_{t}(\alpha)\right|^{2}<\rho^{-(1-\gamma)}\right\} \\
& \doteq \operatorname{Pr}\left\{\left|\prod_{t=1}^{T} H_{t}\left(n_{m}\right)\right|^{2}<\rho^{-(1-\gamma)}, m=G, \ldots, K\right\} \\
& \doteq \operatorname{Pr}\left\{\sum_{m=G}^{K}\left|\prod_{t=1}^{T} H_{t}\left(n_{m}\right)\right|^{2}<\rho^{-(1-\gamma)}\right\} \\
& \leq \operatorname{Pr}\left\{\left|\mathbf{h}_{G}\right|^{2}<\rho^{-(1-\gamma)}\right\}
\end{aligned}
$$

Following similar steps in (21)-(24), we can obtain

$$
\operatorname{Pr}(\mathcal{E}) \dot{\leq} \rho^{-(1-\gamma) \min \left\{K-G+1, L_{\min }\right\}}
$$

Based on (24) and (29), we can conclude that employing a given full column-rank precoder $\mathbf{U} \in \mathcal{C}^{K \times M}$ to implement linear constellation precoding over an arbitrary group of $K \leq N$ tones at the source node and linear ZF detection at the destination node, the DMT of the OFDM-based multihop relaying system is

$d\left(\gamma_{g} ; M ; G ; K\right)= \begin{cases}\left(1-\gamma_{g} / M\right) \min \left\{K-G+1, L_{\min }\right\}, & \gamma_{g} \in[0, M] \\ 0 & , \quad \gamma_{g} \in[M, K]\end{cases}$

Similarly, for the MMSE detector, we have

$$
\begin{aligned}
\operatorname{Pr}(\mathcal{E}) \doteq & \operatorname{Pr}\left\{\bigcup_{k=1}^{K}\left(\mathrm{SNR}_{m}^{\mathrm{MMSE}}<\rho^{\gamma}\right)\right\} \\
= & \operatorname{Pr}\left\{\frac{1}{\max _{m}\left(\left[\left(\mathbf{U}^{H} \mathcal{H}_{g}^{H} \mathcal{H}_{g} \mathbf{U}+\frac{1}{\rho} \mathbf{I}_{M}\right)^{-1}\right]_{m, m}\right)}\right. \\
< & \left.\rho^{-(1-\gamma)}\right\} \\
= & \operatorname{Pr}\left\{\frac{1}{\lambda_{\min }\left\{\left(\mathbf{U}^{H} \mathcal{H}_{g}^{H} \mathcal{H}_{g} \mathbf{U}+\frac{1}{\rho} \mathbf{I}_{M}\right)^{-1}\right\}}<\rho^{-(1-\gamma)}\right. \\
& \left.+\rho^{-1}\right\} \\
= & \operatorname{Pr}\left\{\lambda_{\min }\left\{\mathbf{U}^{H} \mathcal{H}_{g}^{H} \mathcal{H}_{g} \mathbf{U}\right\}<\rho^{-(1-\gamma)}\right\}
\end{aligned}
$$

Following similar steps in (16)-(29), we can write (31) as

$$
\operatorname{Pr}(\mathcal{E}) \doteq \rho^{-(1-\gamma) \min \left\{K-G+1, L_{\min }\right\}}
$$

So, employing a given full column-rank precoder $\mathbf{U} \in$ $\mathcal{C}^{K \times M}$ to implement linear constellation precoding over an arbitrary group of $K \leq N$ tones at the source and linear MMSE detection at the destination, the DMT of the OFDM-based multihop relaying system is the same as (30).

Based on Theorem 1, it is shown obviously that for given multiplexing gain over a group of $K$ tones, the achievable diversity gain by linear detector, i.e., linear ZF detector or MMSE detector, is decided by $L_{\min }, G$, and $M$. $L_{\min }$ is the smallest channel order over $T$ hop multipath channels, and becomes the performance bottleneck in the high-SNR regime. $G$ is characterized by the full column-rank precoder $\mathbf{U} \in \mathcal{C}^{K \times M}$, so, optimal precoder design is critical of the achievable diversity gain. Furthermore, the value of $M$ denotes the number of transmitted information symbols per group of $K$ tones, which means the symbol loading, and can also be optimized to improve 
the diversity performance of the OFDM-based multihop relaying systems.

\section{Optimal transmit strategies design}

For a given $M$, we see from (13) that the value of $G$ (which is determined by the $K \times M$ precoder) can be optimized to improve the diversity order at any multiplexing gain.

$$
d\left(\gamma_{g} ; M ; K\right)=\max _{M \leq G \leq K} d\left(\gamma_{g} ; M ; G ; K\right), \gamma_{g} \in[0, K]
$$

Notice that if $K-G+1 \geq L_{\min }, \quad d\left(\gamma_{g} ; M ; K\right)=$ $\left(1-\gamma_{g} / M\right) L_{\min }$, which means $M \leq G \leq K-$ $L_{\min }+1$; if $K-G+1 \leq L_{\min }, d\left(\gamma_{g} ; M ; K\right)=$ $\left(1-\gamma_{g} / M\right) \max _{M \leq G \leq K}\{K-G+1\}=\left(1-\gamma_{g} / M\right)(K-$ $M+1)$ when $G=M$. So, we can obtain directly the necessary and sufficient condition of optimal precoder design: For a given $M$, a precoder $\mathbf{U}$ can maximize the diversity order at any multiplexing gain if and only if

$$
M \leq G \leq \max \left\{M, K-L_{\min }+1\right\}
$$

Then, the DMT of the OFDM-based multihop relaying system employing optimal linear constellation precoder over an arbitrary group of $K \leq N$ tones at the source and linear detection at the destination is

$d\left(\gamma_{g} ; M ; K\right)= \begin{cases}\left(1-\gamma_{g} / M\right) \min \left\{K-M+1, L_{\min }\right\} & , \gamma_{g} \in[0, M] \\ 0 & , \gamma_{g} \in[M, K]\end{cases}$

Choosing a precoder with $G=M$ is clearly sufficient to achieve (33). Fortunately, selecting the first $M$ columns of the $K \times K$ DFT matrix [12] (or the rotated signal constellation matrix [10]) as the precoder always yields.

Notice that the symbol loading strategy, which means the value of $M$ itself, can also be optimized. In order to maximize the achievable diversity gain at any multiplexing gain $\gamma_{g}$, we obtain

$$
d\left(\gamma_{g} ; K\right)=\max _{1 \leq M \leq K} d\left(\gamma_{g} ; M ; K\right), \gamma_{g} \in[0, K]
$$

It is clear that $d\left(\gamma_{g} ; K-L_{\min }+1 ; K\right) \geq d\left(\gamma_{g} ; M ; K\right)$ if $M \leq K-L_{\min }+1$. So, we only need to examine the case when $M \geq K-L_{\min }+1$. Further, notice that the diversity order corresponding to $M$ and $M+1$ symbols should cross over at some $\gamma_{g}$. For all multiplexing gains less than that cross-over point, $d\left(\gamma_{g} ; M ; K\right)$ will dominate whereas for all multiplexing gains greater than the cross-over point, $d\left(\gamma_{g} ; K-L_{\min }+1 ; K\right)$ will dominate. To determine the cross-over point, we equate $d\left(\gamma_{g} ; K-\right.$ $\left.L_{\min }+1 ; K\right)=d\left(\gamma_{g} ; M ; K\right)$ and obtain the crossover point to be $M(M+1) /(K+1)$. Similarly, the cross-over point between $d\left(\gamma_{g} ; M+1 ; K\right)$ and $d\left(\gamma_{g} ; M+2 ; K\right)$ can be determined to be $(M+1)(M+2) /(K+1)$. Thus, for $\gamma_{g} \in[M(M+1) /(K+1),(M+1)(M+2) /(K+1)]$, the optimal symbol loading is $M+1$, i.e., in this range $d\left(\gamma_{g} ; M+1 ; K\right)$ dominates all other diversity orders. So, we can obtain the optimal symbol loading strategy over an arbitrary group of $K \leq N$ tones at the source as

$$
M=\left\{\begin{array}{l}
\vartheta, \gamma_{g} \in[0,(\vartheta+1) f(\vartheta)] \\
i, \gamma_{g} \in[(i-1) f(i),(i+1) f(i)], \vartheta+1 \leq i \leq K
\end{array}\right.
$$

where $\vartheta \triangleq \max \left\{K-L_{\min }+1,1\right\}$ and $f(x) \triangleq x /(K+1)$.

Then, we obtain the DMT relation of the OFDM-based multihop relaying system employing the optimal linear constellation precoder and optimal symbol loading strategy over an arbitrary group of $K \leq N$ tones at the source and linear detection at the destination

$d\left(\gamma_{g} ; K\right)=\left\{\begin{array}{c}\left(1-\gamma_{g} / \vartheta\right)(K-\vartheta+1), \gamma_{g} \in[0,(\vartheta+1) f(\vartheta)] \\ \left(1-\gamma_{g} / M\right)(K-M+1), \gamma_{g} \in[(M-1) f(M), \\ (M+1) f(M)], \vartheta+1 \leq M \leq K\end{array}\right.$

Specially, if choosing $M=K$, which means the system employs full-rate symbol loading strategy over an arbitrary group of $K \leq N$ tones, and the precoder is also full-rate, the DMT of the OFDM-based multihop relaying system employing linear constellation precoding and linear detection is

$$
d\left(\gamma_{g} ; K\right)=\left(1-\gamma_{g} / K\right), \gamma_{g} \in[0, K]
$$

It indicates that different from the ML detection, for the OFDM-based relaying system with linear detection, no diversity order gain is achieved when full-rate precoders are employed. The loss of multipath diversity is the price paid for OFDM's simplicity of the linear detection since each symbol is transmitted over a single flat subchannel.

\section{Simulation results and discussion}

In this section, some simulation results are given to validate the theoretical conclusions. We assume that the source node, relay nodes, and destination node are located in a line, and all the relay nodes are equally spaced. The channel between terminals $t-1$ and $t(t \in\{1, \ldots, T\})$ are assumed to be frequency selective Rayleigh fading with the channel order $L_{t}$. We assume a uniform power delay profile, where all taps are subject to Rayleigh fading and path-loss, with the same path loss exponent. All relays work in full-duplex mode and employ the AF protocol to relay the received signals. The channel fading coefficients are assumed to be constant within a OFDM block, and change independently from block to block. Without loss of generality, we set the fixed amplified gain $\alpha=1$. In our simulations, we consider a 3-hop relay network where $T=3$ and the channel order of each hop link is $L_{1}=4, L_{2}=5$, and $L_{3}=6$, respectively. 
In Figure 2, we plot the DMT relation of the OFDMbased multihop AF relaying system employing subcarrier grouping with arbitrary group size $K=6$, linear constellation precoding with deterministic symbol loading $M=5$ at the source node, and linear detector at the destination node with different channel setting, including that Case 1: $L_{1}=3, L_{2}=1, L_{3}=4$, Case $2: L_{1}=3, L_{2}=3, L_{3}=4$, Case 3: $L_{1}=5, L_{2}=5, L_{3}=6$. For the sake of comparison, we also plot the corresponding DMT curves obtained with ML detection. From Figure 2, we can find that both linear detection and ML detection can only achieve the diversity gain of 1 when the multiplexing gain $\gamma_{g}=0$ in Case 1 because the smallest of the multipath channel orders is $L_{2}=1$, which is the performance bottleneck in the high-SNR regime in Case 1. Furthermore, there is the same DMT curve for the OFDM-based multihop AF relaying system when employing linear detection in Cases 2 and 3 . Because, in these cases, $K-M+1<L_{\min }$ and the achievable diversity gain is determined by the relationship between $K$ and $M$ based on Theorem 1 .

In Figure 3, we plot the DMT relation of the OFDMbased multihop AF relaying system employing subcarrier grouping with arbitrary group size $K=6$, linear constellation precoding with different symbol loading at the source node, and linear detector at the destination node. For the sake of comparison, we also plot the corresponding DMT curves obtained with ML detection. In this simulation, we set $L_{1}=4, L_{2}=5, L_{3}=6$, and employ the optimal symbol loading strategy based on (37). From Figure 3, the optimal symbol loading strategy provides significant diversity gains over the conventional loading, particularly at lower multiplexing gain. However, for linear detection, no diversity gain is achieved when the full-rate precoder with maximal symbol loading, $K=M=\gamma_{g}$, is employed.

In Figure 4, we plot the DMT relation of the OFDMbased multihop AF relaying system employing subcarrier grouping with different group size $K=4,5,6,8$, linear constellation precoding with optimal symbol strategy based on (37) at the source node, and linear detector at the destination node. Figure 4 indicates that the achievable diversity gain is improved with the increase of the group size $K$ at the given multiplexing gain. However, a large value of $K$ would result in the increase of the linear detection complexity.

In Figure 5, we plot the outage probability versus SNR curves for the OFDM-based multihop AF relaying system employing subcarrier grouping, linear constellation precoding with different symbol loading at the source node, and linear ZF detector at the destination node. We consider an OFDM-based relaying system with $N=32$ tones out of which a group of $K=8$ tones are used for data transmission, and the given threshold rate is $1 \mathrm{bit}$ per-channel-use, which mains that 1 bit information per symbol is transmitted over each tone in the group. The data symbols are precoded using a precoder formed by the first $M$ th columns of a $K \times K$ DFT matrix. For the sake of comparison, we also plot the corresponding outage probability curves obtained with ML detection. Note that the case of $M=8$ means a full-rate precoder employed. Because of full-rate precoder in the case, the ML detector can achieve full multipath diversity with a diversity order of 4 whereas the linear detectors have a diversity order of

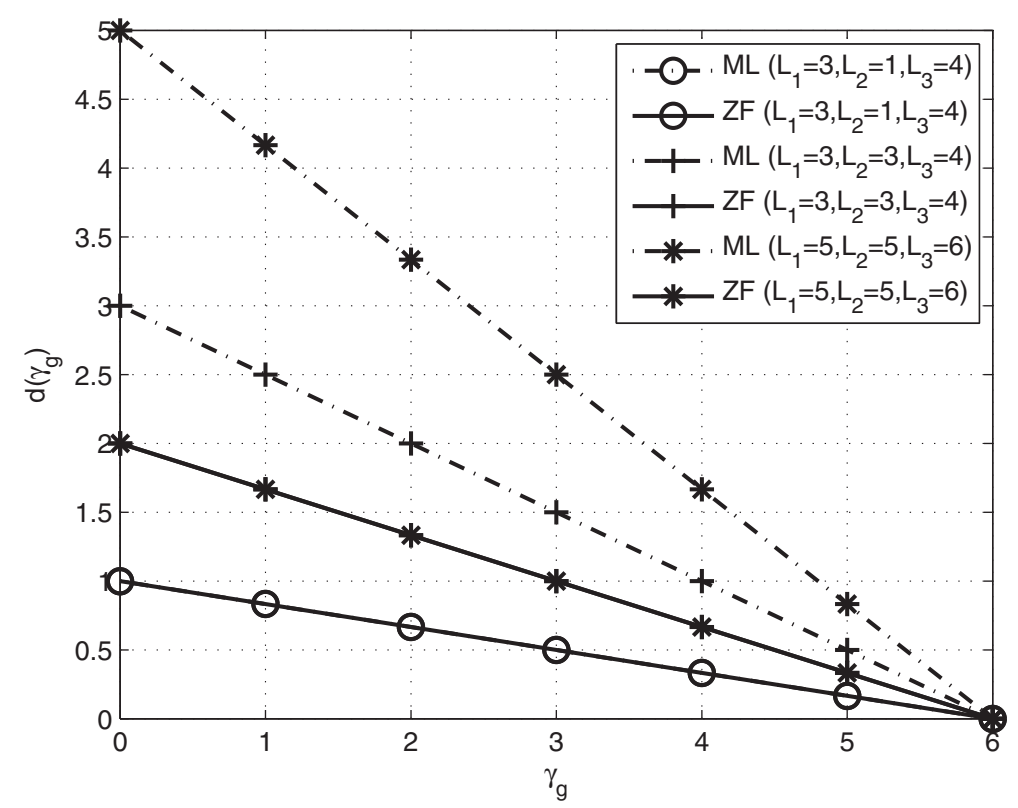

Figure 2 DMT of the OFDM-based multihop AF relaying system with different channel setting $(K=6$ and $M=5)$. 


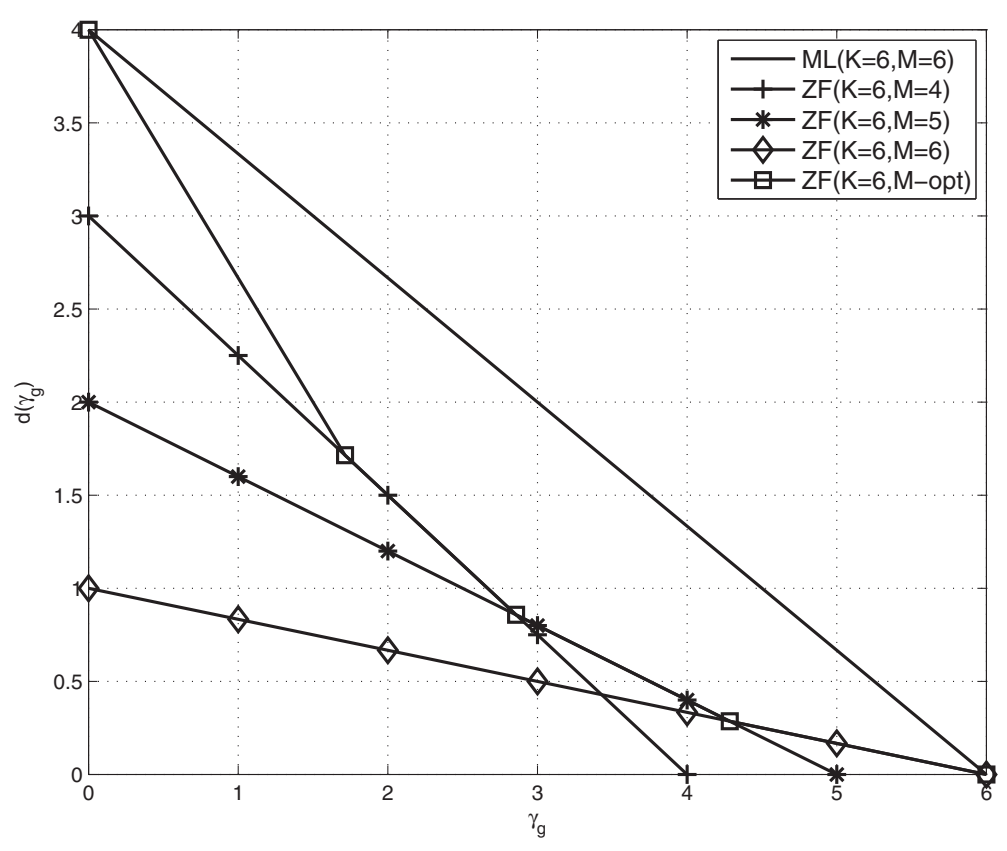

Figure 3 DMT of the OFDM-based multihop AF relaying system with different symbol loading $\left(K=6\right.$ and $L_{1}=4, L_{2}=5, L_{2}=6$ ).

1. With the decrease of $M$, the achievable diversity gain is improved for linear detection, and the slope of the curves become more and more sharp. It can be also observed that the curves of $M=5$ and $M=3$ for linear detection both have the identical slope, especially in high SNR, due to the same achievable diversity gain. On the other hand, for ML detection, full multipath diversity gain can always be achieved with different $M$, the curves have the identical slope.

In Figure 6, we plot the outage probability versus SNR curves for the OFDM-based multihop AF relaying system employing different precoding design at the source node and linear ZF and MMSE detector at the destination node. We consider the same set-up as in Figure 5,

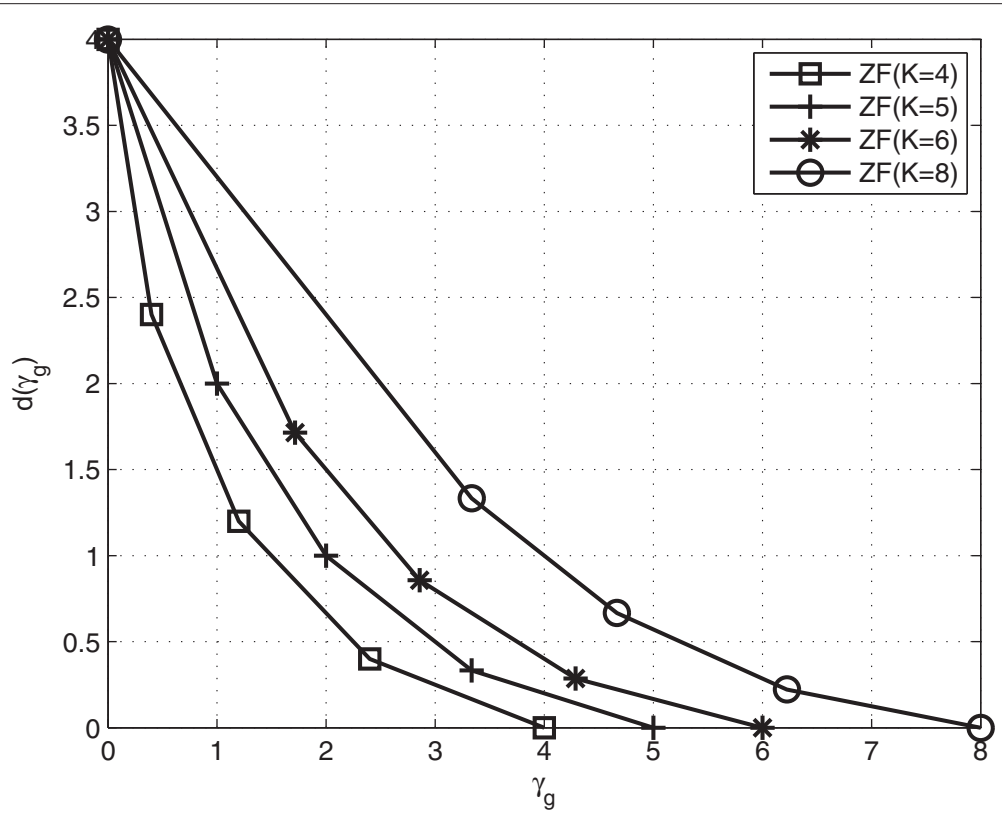

Figure 4 DMT of the OFDM-based multihop AF relaying system with different group size $\left(L_{1}=4, L_{2}=5, L_{3}=6\right)$. 


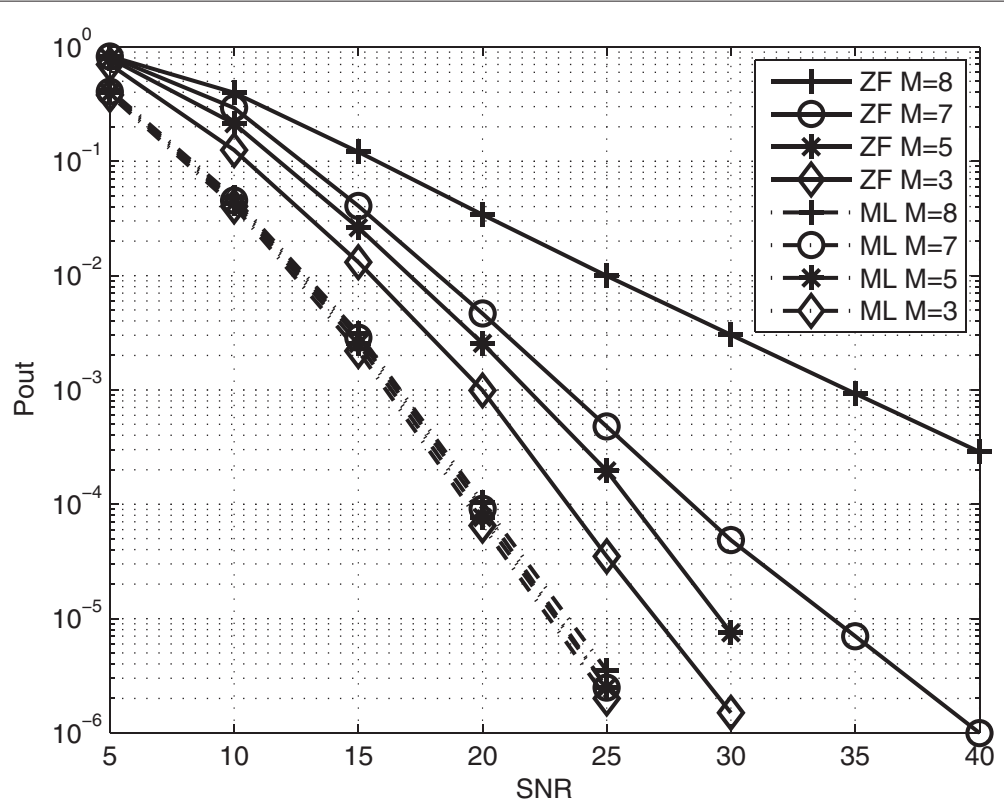

Figure 5 Outage probability versus SNR curves for different symbol loading.

besides three different precoders as: (1) non-precoding scheme which means the source node do not precode the data symbols, (2) DFT-precoding scheme which means the data symbols are precoded using a precoder formed by the first $M$ th columns of a $K \times K$ DFT matrix, (3) RSC-precoding scheme which means the data symbols are precoded using a precoder formed by the first $M$ th columns of a $K \times K$ rotated signal constellation matrix base on [10]. It can be seen from Figure 6 that the outage performance with linear constellation precoding is improved significatively compared to that without precoding. Note that as predicted by our analysis, the outage performance curves employed DFT-precoding and RSC-precoding has the identical slope in high-SNR due to the same achievable diversity gain. In can also observed that the MMSE detector exhibits a much better

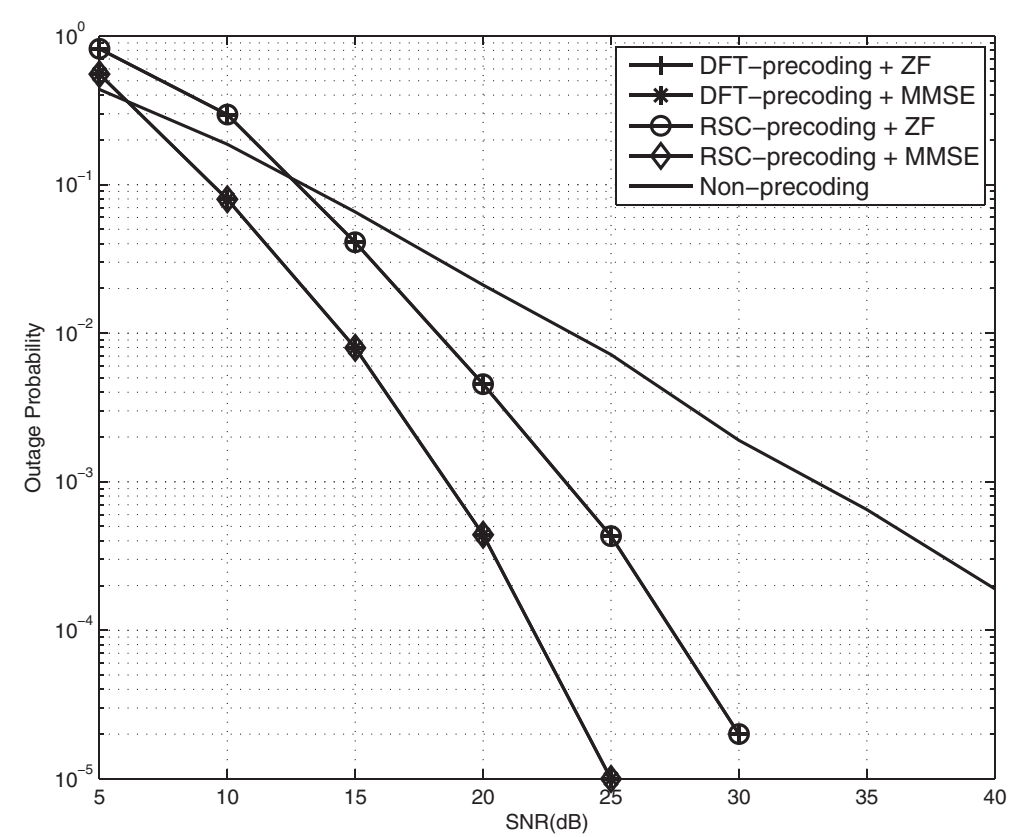

Figure 6 Outage probability versus SNR curves for different precoder design. 
performance for a wide range of SNRs compared to the ZF detector.

\section{Conclusions}

In this study, we have derived the closed-form expressions of the DMT relation for OFDM-based multihop AF relaying systems employing subcarrier grouping and linear constellation precoding at the source node, and linear detection at the destination node. The results show that the smallest of the multipath channel orders experienced in each wireless links becomes the performance bottleneck in the high-SNR regime, and the achievable multipath diversity gain by linear detectors is dependent upon the symbol loading and precoder design. Then, the optimal precoder and the symbol-loading strategy have been proposed to achieve optimal diversity performance. Accordingly, a complete analysis and optimization framework for OFDM-based multihop relaying systems over frequency selective fading channels has been obtained, which can be used as a powerful tool for performance evaluation and optimal design of existing and upcoming OFDM-based multihop relaying systems.

\section{Competing interests}

The authors declare that they have no competing interests.

\section{Acknowledgements}

This study was supported by the NSF of China (Grant No. 61001107 , 60972051), the Major National Science \& Technology Projects (Grant No. 2010ZX03006-002-04), and the Jiangsu Natural Science Foundation (Grant No. BK2010101).

\section{Author details}

${ }^{1}$ Institute of Communications Engineering, PLA University of Science and Technology, Nanjing, China. ${ }^{2}$ Nanjing University of Posts and Telecommunication, Nanjing, China.

Received: 16 December 2011 Accepted: 20 July 2012

Published: 7 August 2012

\section{References}

1. O Oyman, JN Laneman, S Sandhu, Multihop relaying for broadband wireless mesh networks: From theory to practice. IEEE Commun. Mag. 17, 116-122 (2007)

2. K Sreeram, S Birenjith, PV Kumar, DMT of multi-hop cooperative networks—Part I: bsic results [Online] http://arxiv.org/abs/0808.0234

3. K Sreeram, S Birenjith, PV Kumar, DMT of multi-hop cooperative networks - Part II: half-duplex networks with full-duplex performance. [Online] http://arxiv.org/abs/0808.0235

4. Z Wang, G Giannakis, Wireless multicarrier communications: where Fourier meets Shannon. IEEE Signal Process. Mag. 17, 29-48 (2000)

5. IEEE 802.16's Relay Task Group. [Online] http://www.ieee802.org/16/relay/

6. B Can, M Portalski, HSD Lebreton, S Frattasi, HA Suraweera, Implementation issues for OFDM-based multihop cellular networks. IEEE Commun. Mag. 17, 74-81 (2007)

7. L Song, M Tao, $Y X u$, Exploiting hop diversity with frequency sharing in multi-hop OFDM networks. IEEE Commun. Lett. 13, 908-910 (2009)

8. X Zhang, M Tao, W Jiao, S Chun, End-to-end outage minimization in OFDM based linear relay net- works. IEEE Trans. Commun. 57, 3034-3044 (2000)

9. Z Liu, Y Xin, G Giannakis, Linear constellation precoding for OFDM with maximum multipath diversity and coding gains. IEEE Trans. Commun. 51, 416-427 (2003)
10. W Zhang, Y Li, X-G Xia, PC Ching, BL Khaled, Distributed space-frequency coding for cooperative diversity in broadband wireless ad hoc networks. IEEE Trans. Wirel. Commun. 7, 995-1003 (2008)

11. L Venturino, N Prasad, $X$ Wang, M Madihian, Design of linear dispersion codes for practical MIMO- OFDM systems. IEEE J. Sel. Topics Signal Process. 1, 178-188 (2007)

12. CTepedelenlioglu, Maximum multipath diversity with linear equalization in precoded OFDM systems. IEEE Trans. Inf. Theory. 50, 232-235 (2004)

13. L Zheng, DNC Tse, Diversity and multiplexing: a fundamental tradeoff in multiple-antenna channels. IEEE Trans. Inf. Theory. 49, 1073-1096 (2003)

14. Sh Yang, J-C Belfiore, Optimal space-time codes for the MIMO amplify-and-forward cooperative chan- nel. IEEE Trans. Inf. Theory. 52, 647-663 (2006)

15. M Yuksel, E Erkip, Multiple-antenna cooperative wireless systems: a diversity-multiplexing tradeoff perspective. IEEE Trans. Inf. Theory. 53, 3371-3393 (2007)

16. AA Haghighi, K Navaie, Outage analysis and diversity-multiplexing tradeoff bounds for opportunistic relaying coded cooperation and distributed space-time coding coded cooperation. IEEE Trans. Wirel. Commun. 9, 1198-1206 (2010)

17. O Leveque, C Vignat, M Yuksel, Diversity-multiplexing tradeoff for the MIMO static half-duplex relay. IEEE Trans. Inf. Theory. 56, 3356-3368 (2010)

18. S Yang, J-C Belfiore, Diversity of MIMO multihop relay channels. IEEE Trans. Inf. Theory. http://www.ieee802.org/16/relay/

19. D Gunduz, A Khojastepour, A Goldsmith, HV Poor, Multihop MIMO relay networks: diversity- multiplexing trade-off analysis. IEEE Trans. Wirel. Commun. 9, 1198-1206 (2009)

20. Q Li, KH Li, KC Teh, Achieving optimal diversity-multiplexing tradeoff for full-duplex MIMO multihop relay networks. IEEE Trans. Inf. Theory. 57, 303-316 (2011)

21. L Grokop, DNC Tse, in Proc. 2007 IEEE Int. Symp. Information Theory (ISIT) Diversity-multiplexing tradeoff in ISI channel. (Chicago, IL, 2004), pp. 96

22. L Grokop, DNC Tse, Diversity-multiplexing tradeoff in ISI channel. IEEE Trans. Inf. Theory. 55, 109-135 (2009)

23. A Medles, DTM Slock, in Proc. 2007 IEEE Int. Symp. Information Theory (ISIT) Optimal diversity vs. multiplexing tradeoff for frequency selective MIMO channels. (Adelaide, Australia, 2005), pp. 1813-1817

24. P Coronel, H Bolcskei, in Proc. 2007 IEEE Int. Symp. Information Theory (ISIT) Diversity-multiplexing tradeoff in selective-fading MIMO channels. (Nice, France, 2007), pp. 2841-2845

25. N Prasad, L Venturino, $X$ Wang, Diversity-multiplexing tradeoff analysis for OFDM systems with sub- carrier grouping, linear precoding, and linear detection. IEEE Trans. Inf. Theory. 56, 6078-6096 (2010)

26. Y Jiang, MK Varanasi, I Li, Performance analysis of ZF and MMSE equalizers for MIMO systems: an indepth study of the high SNR regime. IEEE Trans. Inf. Theory. 57, 2008-2026 (2011)

27. RA Horn, CR Johnson, Matrix Analysis (Cambridge, U.K., Cambridge Univ. Press, 1993)

doi:10.1186/1687-1499-2012-245

Cite this article as: Yang et al:: Diversity-multiplexing tradeoff analysis and optimization for OFDM-based multihop relaying systems. EURASIP Journal on Wireless Communications and Networking 2012 2012:245. 